\title{
Reaction forces and bone maturation in taekwondo: comparison of practice with and without tatami
}

original paper

() Wroclaw University of Health and Sport Sciences

DOI: https://doi.org/10.5114/hm.2023.107246

\author{
FERNANDO ROCHA ${ }^{1 \oplus}$, ANA CONCEIÇÃO ${ }^{1,2 \oplus}{ }^{\oplus}$ CARLOS MATA $^{1,3 \oplus}$, JANINE NARCISO $^{2 \oplus}$, \\ ALDO M. COSTA ${ }^{2,4,5 \oplus, ~ M A R C O ~ B R A N C O ~}{ }^{1 \oplus}$, HUGO LOURO $^{1,2 \oplus}$ \\ ${ }^{1}$ Sport Sciences School of Rio Maior, Rio Maior, Portugal \\ ${ }^{2}$ Research Center in Sports Sciences, Health Sciences and Human Development (CIDESD), Vila Real, Portugal \\ ${ }^{3}$ Life Quality Research Centre, Rio Maior, Portugal \\ ${ }^{4}$ Department of Sport Sciences, University of Beira Interior, Covilhã, Portugal \\ ${ }^{5}$ Health Sciences Investigation Center, University of Beira Interior, Covilhã, Portugal
}

\begin{abstract}
Purpose. Exercise performed improperly, in disagreement with age and development, may trigger negative bioactive effects. Adolescent taekwondo athletes, being able to practise on tatami or without tatami and barefoot, may be submitted to musculoskeletal structure disorders. The purpose of this study was to describe the frequency and amplitude of the reactive forces during taekwondo practice with and without tatami.

Methods. Overall, 27 adolescent taekwondo athletes aged 10-15 years were submitted to a standard 90-minute training session with and without tatami. During each session, the frequency of ground contacts and the vertical ground reaction forces were measured. Femur and wrist bone age and the respective stages of the epiphyseal plate were computed from radiograph images. A total of $6346.6 \pm 425.5$ ground contacts (an average of 106 contacts per minute) were registered during the training session. The Wilcoxon test was applied to analyse the relationship between taekwondo and epiphyseal plate stage $(p \leq 0.005)$.

Results. Student's $t$-test revealed a significant difference between ground reaction forces with and without tatami; tasks performed without tatami featured smaller magnitudes for comparisons of absolute and normalized values $(t=-3.359$; $p<0.002$ and $t=-3.652 ; p<0.001$, respectively). The time of practice showed no early closure of the femur epiphyseal plates caused by the practice of taekwondo.
\end{abstract}

Conclusions. Practising taekwondo seems to have a bio-positive effect on the maturation of adolescent athletes, regardless of the use of tatami.

Key words: taekwondo, support reaction forces, epiphyseal growth plates, bone maturation, adolescents

\section{Introduction}

Many children and adolescents start their sports training early in their lives, which often leads to an early specialization, with several weekly workouts and a high number of hours at a level of intensity necessary to be competitive. In taekwondo, increasing numbers of practitioners have directed greater attention to safety and injury identification needs, towards a safe practice. However, there is also a rise in the heterogeneity of the classes of practitioners, which seems to have resulted in a higher incidence of injuries, particularly among children [1].

Since the inclusion of taekwondo in the Olympic Games, there have been several changes in terms of rules and regulations, safeguarding the integrity of the sport. In 1986, the Kukkiwon, also known as the World Taekwondo Headquarters, adopted the ethylene-vinyl acetate (EVA) floor (flexible tatami) in official competitions, equalling the attitude taken previously by judo [2]. This floor is designed with the goal of providing better comfort by minimizing the possi-

Correspondence address: Ana Conceição, Sports Sciences School of Rio Maior, Polytechnic Institute of Santarém, Av. Dr. Mário Soares, 2040-413 Rio Maior, Portugal, e-mail: anaconceicao@esdrm.ipsantarem.pt, https://orcid.org/0000-0002-8109-4875

Received: February 4, 2021

Accepted for publication: May 11, 2021

Citation: Rocha F, Conceição A, Mata C, Narciso J, Costa AM, Branco M, Louro H. Reaction forces and bone maturation in taekwondo: comparison of practice with and without tatami. Hum Mov. 2023;24(1):76-85; doi: https://doi.org/10.5114/ hm.2023.107246. 
bility of injury. In fact, the regular practice of this sport necessarily promotes micro-injuries by the constant contact with the ground (ground reaction forces [GRF]). About $75 \%$ of the mechanical energy of an impact can be passively dissipated through muscles, joints, and ligaments, while the remaining $25 \%$ will dissipate through other structures, such as footwear, pavement, etc. [3].

The Apkubi taekwondo technique analysis showed 1.4 body weight (BW) vertical reactions forces, a smaller value than the one reported for walk activity, $1.8 \mathrm{BW}$ $(t=6.085 ; p<0.001)$. This peak is registered when receiving or contacting the foot with the ground, which constitutes an important phase of deceleration for the structures in sports movements and is identified with a considerable mechanical overload applied to the locomotor system [4]. In the evaluation of 10 experienced capoeiristas, the values found were $1.36 \pm 0.17 \mathrm{BW}$ for the movement called the hammer and $1.91 \pm$ $0.36 \mathrm{BW}$ for the closed negative movement; values of $5.15 \pm 2.06 \mathrm{BW}$ for the screw movement and $4.67 \pm$ 1.65 BW for the skipped armed movement were also registered [5]. The magnitude of GRFs in terms of \% BW in the rotating kick in taekwondo evaluated with a force platform from 3 positions relative to a fixed target is as follows: in the $0^{\circ}$ position, the result was $4 \mathrm{BW}$, being the highest peak at the front foot; at the $45^{\circ}$ position, the magnitude of GRF was evenly distributed; at the last $90^{\circ}$, the magnitude of GRF was 4 BW, with the highest peak at the rear foot [6].

In general, almost every contact sports activity produces GRF, and the impact is expected to have a biopositive effect when it occurs in a range of safety, promoting the osteoblastic activity in the bone region near the places where muscles are inserted, leading to an increase of the bone mineralization. On the other hand, both absences of muscle contraction (due to immobilization or fractures) and excess impact beyond the physiological limits of tolerance may inhibit the growth and normal development of the young athlete [7]. Thus, the tolerance limits of the epiphysis plate are a matter of concern of the technical and scientific community [8]. The joint impact resulting from systematic training and exercises that make up the routine of young athletes have been mainly discussed in the context of a potential damage to the epiphysis plate, hindering the normal process of bone growth [9]. In fact, some recent findings suggest a cam-type abnormality in elite basketball players as a consequence of an alteration of the growth plate rather than reactive bone formation [10]. However, significant gains were also reported in bone mineral content and bone area at the femoral neck resulting from 7 months of high-impact training in prepubertal children; these gains were retained after an equivalent period of detraining [11]. Therefore, the scientific evidence is not entirely sound on this subject, and it is absolutely imperative to encourage the scientific community to further study this problem [9].

To our knowledge, there are no studies on this issue in young taekwondo athletes, who experience significant and frequent high impacts over a very short period, especially during training. Thus, the purpose of this study was to describe the frequency and amplitude of the reactive forces during taekwondo practice with and without tatami. Considering the possible effect of GRFs on bone maturation, we also analysed the relationship of this parameter with the femur and wrist bone age and the respective stages of the epiphyseal plate.

\section{Material and methods}

\section{Participants}

A sample of 27 taekwondo adolescent athletes of both genders (age: $12.37 \pm 1.77$ years) volunteered to participate in this study. The inclusion criterion was a minimum of 3 years of continued practice, with at least 3 hours of weekly training. Organized practice of other sports in the previous 3 years was considered an exclusion criterion.

The participants were randomly assigned to a 'tatami' group $(n=13)$ and a 'without tatami' group $(n=14)$. The athletes' federal licence was also verified to attest the absence of any impediment to the practice of taekwondo. All subjects were national level athletes exhibiting, at the time of this study, good overall performance.

The adolescents and their parents were informed in advance about the procedures of the study.

\section{Test protocol}

A cross-sectional research design was selected. The frequency and amplitude of reactive forces were monitored with a Bioplux force platform (Plux wireless biosignals, Lisbon, Portugal) during taekwondo practice with and without tatami. The variables selected were GRF and bone maturation.

The anthropometric assessment was carried out in accordance with the International Working Group of Kinanthropometry recommendations [12]. We evaluated height $(\mathrm{m})$ to the nearest $0.10 \mathrm{~cm}$ with a stadi- 
F. Rocha, A. Conceição, C. Mata, J. Narciso, A.M. Costa, M. Branco, H. Louro, Reaction forces in taekwondo

ometer (SECA, model 225, Germany). Weight was assessed with a Tanita body composition analyser (model TBF-200, Tanita Corporation of America, Inc., Arlington Heights, USA).

GRFs generated by BW were measured with a Bioplux force platform (Plux wireless biosignals, Lisbon, Portugal). The force platform training session protocol involved practice with and without tatami, which consisted in accomplishing displacements, jumps, turns, spins, and stance changes. The force platform was levelled with the floor to walk normally on it. The platform has a square shape $(60 \times 60 \mathrm{~cm})$ and can evaluate the impacts in 3 axes; however, only the values of the vertical impact ( $\mathrm{Z}$ axis) were used (Figure 1 ).

A

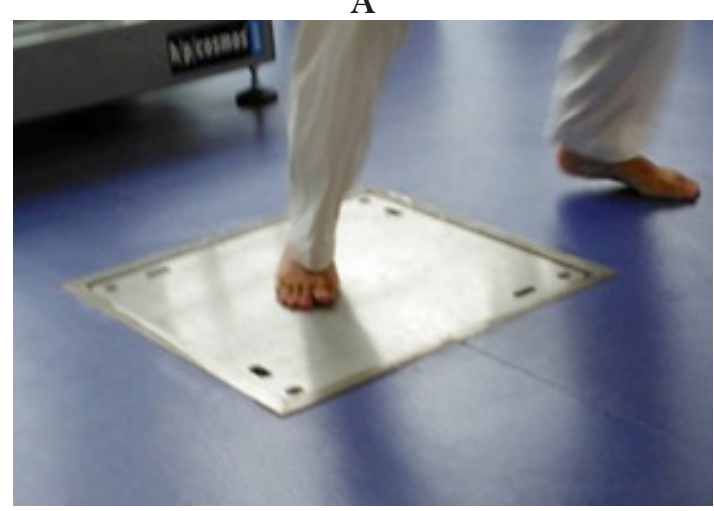

B

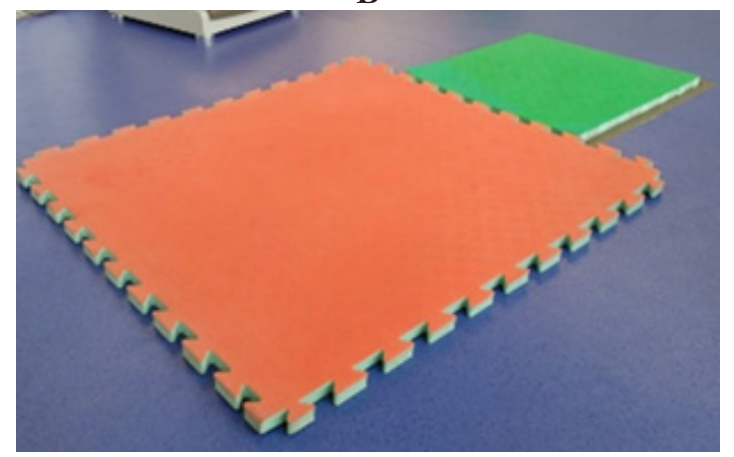

Figure 1. Force platform levelled with the square shape tatami $(60 \times 60 \mathrm{~cm})$. (A) Recessed floor platform.

(B) Placing the tatami for collecting data

The force platform uses a load cell, a strain gauge type transducer, i.e. an electrical device that exhibits electrical resistance as a function of its mechanical deformation [13]. The conversion factor used for newton $(\mathrm{N})$ was 1684 per volt $(\mathrm{V})(1 \mathrm{~V}-1684 \mathrm{~N})$. The data were collected directly to a computer through the OpenSignals (r)evolution program (beta v. 2015, Plux wireless biosignals, Lisbon, Portugal).

To record the number of jumps/impacts performed by the athletes during a typical training session (with and without tatami), we used a Samsung Galaxy S4
GT-I9500 smartphone (Samsung Electronics Co., Ltd., Suwon, South Korea) with the Android-based pedometer application Tayutau. Results from other studies showed that Tayutau produced the lowest mean absolute percent error (6.7\%) and was the only smartphone application at the time with acceptable accuracy in counting steps in a laboratory setting [14].

The medical imaging team from the Santarém District Hospital conducted the radiological investigations for each athlete. The subjects underwent radiography of the left wrist and hand (posterior-anterior view) and of the distal femur epiphyseal plate. We followed the O'Connor et al. [15] protocol, using a SHIMADZU radiographic system. Exposure was obtained on an AGFA cassette and digitalized by AGFA 30-X CR Reader.220. Bone age and the stages of epiphyseal plate were computed by radiologists from the selected radiograph images by using the Greulich and Pyle [16] atlas protocol.

After calculating bone age by hand and wrist radiography, we obtained the indicator of maturity stages using the difference between chronological age and bone age $[16,17]$. For femur epiphyseal plate, we applied the following description stages [16]: 0 - non union; 1 - onset of union; 2 - active union; 3 - recent union; 4 - complete union.

In the laboratory, for GRF evaluation, we used a double-sided tape to clip and secure the tatami plaque with the exact measurement of the force platform and added another plaque to ensure that the athlete was always on the tatami. The protocol followed was as shown in Table 1. Each individual started the protocol with task 1 , and it was only after all participants had gone through this task that task 2 was performed, and so on.

Table 1. Force platform training session protocol

\begin{tabular}{llll}
\hline \multirow{2}{*}{ Condition } & \multicolumn{1}{c}{ Sequence } & Task & $\begin{array}{l}\text { Number of } \\
\text { repetitions }\end{array}$ \\
\hline & $1^{\text {st }}$ & Technical displacements & 5 \\
\cline { 2 - 4 } & $2^{\text {nd }}$ & Competition displacements & 5 \\
\cline { 2 - 4 } $\begin{array}{l}\text { With } \\
\text { and } \\
\text { without } \\
\text { tatami }\end{array}$ & $3^{\text {rd }}$ & Technical jumps & 5 \\
\cline { 2 - 4 } & $4^{\text {th }}$ & Competition jumps & 5 \\
\cline { 2 - 4 } & $5^{\text {th }}$ & Technical turns & 5 \\
\cline { 2 - 4 } & $6^{\text {th }}$ & Competition turns & 5 \\
\cline { 2 - 4 } & $7^{\text {th }}$ & Spins & 5 \\
\hline
\end{tabular}


At the gym, for the frequency of ground contacts evaluation, we based on the characteristics of the taekwondo sport. Three trainers, one $5^{\text {th }}$ Dan black belt rank and two $4^{\text {th }}$ Dan black belt rank, built an 80-minute (28-minute first part: warm-up jogging, jump, taekwondo specific games; 40-minute main part: taekwondo stances, kicking, displacements, poomsae, combat; 12-minute final part: return to calm, moment of meditation and imagery) training session in order to be able to reproduce it in similar conditions. Each exercise contained a description, time, and success criteria, as well as the number of repetitions, number of series, the intensity, and the pause time.

All contacts were made by the feet, without training shoes (barefoot). Before each training unit, we unlocked the smartphone, set it to the maximum sensitivity stage, and then attached it to the athlete's belt with adhesive tape. We started the application simultaneously on all the devices.

During the training unit, we performed 3 stops for data collection. The first collection was at the end of the first phase of training, the second collection at the end of the main phase of training, and the third collection at the end of the final stage of training, which corresponded to the end of the training unit.

The athletes received instructions and demonstration of the tasks that they were to execute. Prior to the start of data collection, each participant rehearsed the protocol in the 2 conditions: with and without tatami.

\section{Statistical analysis}

The Shapiro-Wilk test was used to test the normality of distribution, not assumed in all cases. Student's $t$-test for paired samples served to compare the averages of GRFs with and without tatami. Spearman's correlation was applied to verify the relationship of the reaction forces with the stage of the epiphyseal plate and to check the relationship of GRF magnitudes through years of practice.

To verify the existence of differences in the average age and the stages of distal femoral epiphyseal plate between boys and girls, i.e. to check if the taekwondo athletes had a different maturation status, the MannWhitney $U$ test was applied for 2 independent samples. The Wilcoxon test was used in the analysis of the relationship between taekwondo practice time and the stage of the epiphyseal plate. Statistical analysis was carried out with the IBM SPSS software, version 23. Statistical significance was established at the level of $p<0.05$.

\section{Ethical approval}

The research related to human use has complied with all the relevant national regulations and institutional policies, has followed the tenets of the Declaration of Helsinki, and has been approved by the authors' institutional review board or an equivalent committee.

\section{Informed consent}

Informed consent has been obtained from all individuals included in this study and their legal guardians.

\section{Results}

Table 2 presents the normative data for the sample, and Table 3 depicts the contacts made in the context of training and GRFs in newtons (N) and normalized to BW.

It was revealed that a standard 60-minute taekwondo class involved an average of 6347 contacts, about 106 contacts per minute. These values are in the frequency range indicated for the bone bio-positive effects. The tasks performed without tatami featured smaller magnitudes of absolute GRF when compared with the tasks performed with tatami, with an average difference of about $10 \%$ of BW $(t=-3.359 ; p<0.002$ and $t=-3.652 ; p<0.001$, respectively, for comparisons of absolute and normalized values).

Considering the possible effect that GRF has on bone maturation, we analysed the association between GRFs and the epiphyseal stage for the wrist and femur in the 2 implemented conditions: with tatami and without tatami (Table 4).

The results show that there was a positive correlation between the variables of maturation and GRF in the performed tasks. This correlation seems to be stronger for the GRF without tatami. For the entire sample, there was a significant correlation between the variables and decimal age, independently of the floor type.

With regard to the possible effect that the practice of taekwondo has on the magnitude of the absolute GRF, there was a moderate positive correlation $(\rho=$ $0.382 ; p<0.05$ ) between the years of practice and the GRF of tasks performed without tatami and also a moderate positive correlation $(\rho=0.410 ; p<0.05)$ with the GRF of tasks performed with tatami.

The trendline of $\mathrm{R}^{2}$ of $12 \%$ and $14 \%$ of GRF with tatami and without tatami, respectively, is due to the years of taekwondo practice, that is, by the trend, larger magnitudes of GRF were observed with more years of practice (Figure 2). 


\section{HUMAN MOVEMENT}

F. Rocha, A. Conceição, C. Mata, J. Narciso, A.M. Costa, M. Branco, H. Louro, Reaction forces in taekwondo

Table 2. Normative data (mean $\pm S D)$

\begin{tabular}{lccccc}
\hline Characteristics & $n$ & Height $(\mathrm{m})$ & Weight $(\mathrm{kg})$ & Practice time (years) & Weekly training (hours) \\
\hline Males & 19 & $1.56 \pm 0.13$ & $52.79 \pm 15.17$ & $4.24 \pm 1.85$ & $4 \pm 0.92$ \\
Females & 8 & $1.53 \pm 0.06$ & $47.78 \pm 7.79$ & $3.75 \pm 1.23$ & $3.93 \pm 0.85$ \\
With tatami & 13 & $1.55 \pm 0.11$ & $50.88 \pm 14.58$ & $4.15 \pm 1.81$ & $4.07 \pm 0.98$ \\
Without tatami & 14 & $1.56 \pm 0.12$ & $51.73 \pm 12.66$ & $4.04 \pm 1.60$ & $3.88 \pm 0.81$ \\
Total & 27 & $1.55 \pm 0.11$ & $51.30 \pm 13.54$ & $4.09 \pm 1.69$ & $3.98 \pm 0.90$ \\
\hline
\end{tabular}

Table 3. Descriptive statistics of the contacts and ground reaction forces

\begin{tabular}{lccccc}
\hline \multirow{2}{*}{ Data } & \multirow{2}{*}{$\begin{array}{c}\text { Number } \\
\text { of contacts }\end{array}$} & \multicolumn{2}{c}{ GRF (BW) } & \multicolumn{2}{c}{ Absolute GRF (N) } \\
\cline { 3 - 6 } & \multirow{2}{*}{ With tatami } & Without tatami & With tatami & Without tatami \\
\hline Minimum & 5321.0 & 1.7 & 1.7 & 526.6 & 479.8 \\
Maximum & 7073.0 & 2.7 & 2.6 & 1829.6 & 1827.3 \\
Mean $\pm S D$ & $6346.6 \pm 425.5$ & $2.1 \pm 0.2$ & $2.0 \pm 0.2$ & $1070.8 \pm 276.8$ & $1025.3 \pm 280.2$ \\
\hline
\end{tabular}

GRF - ground reaction forces, BW - body weight

Table 4. Spearman correlation of the variables of ground reactions forces and decimal age with bone age and femoral and wrist epiphyseal stage, per surface/floor

\begin{tabular}{|c|c|c|c|c|c|c|}
\hline Variable & Floor & & $\begin{array}{l}\text { Ground reaction } \\
\text { force }\end{array}$ & $\mathrm{R}^{2}$ & Decimal age & $\mathrm{R}^{2}$ \\
\hline \multirow{2}{*}{ Bone age } & With tatami & $\begin{array}{c}\rho \\
\text { Significance }\end{array}$ & $\begin{array}{r}0.688 \\
<0.001\end{array}$ & 0.47 & $\begin{array}{r}0.829 \\
<0.001\end{array}$ & 0.69 \\
\hline & Without tatami & $\begin{array}{c}\rho \\
\text { Significance }\end{array}$ & $\begin{array}{r}0.763 \\
<0.001\end{array}$ & 0.58 & $\begin{array}{r}0.844 \\
<0.001\end{array}$ & 0.71 \\
\hline \multirow{2}{*}{ Femur epiphyseal stage } & With tatami & $\begin{array}{c}\rho \\
\text { Significance }\end{array}$ & $\begin{array}{r}0.522 \\
<0.001\end{array}$ & 0.27 & $\begin{array}{r}0.878 \\
<0.001\end{array}$ & 0.77 \\
\hline & Without tatami & $\begin{array}{c}\rho \\
\text { Significance }\end{array}$ & $\begin{array}{r}0.607 \\
<0.001\end{array}$ & 0.37 & $\begin{array}{r}0.691 \\
<0.001\end{array}$ & 0.47 \\
\hline \multirow{2}{*}{ Wrist epiphyseal stage } & With tatami & $\begin{array}{c}\rho \\
\text { Significance }\end{array}$ & $\begin{array}{r}0.507 \\
<0.001\end{array}$ & 0.26 & $\begin{array}{r}0.802 \\
<0.001\end{array}$ & 0.64 \\
\hline & Without tatami & $\begin{array}{c}\rho \\
\text { Significance }\end{array}$ & $\begin{array}{r}0.609 \\
<0.001\end{array}$ & 0.38 & $\begin{array}{r}0.625 \\
<0.001\end{array}$ & 0.39 \\
\hline
\end{tabular}

Significance at $p<0.05$
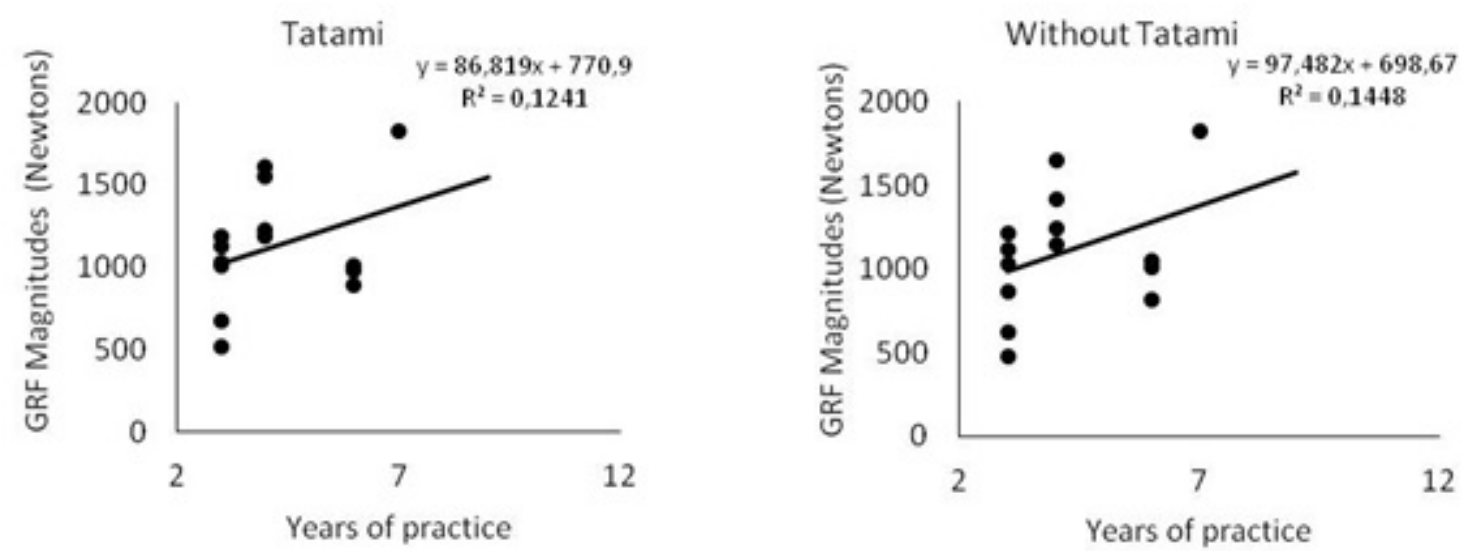

Figure 2. Trend line between the years of taekwondo practice and the magnitudes of absolute GRF for the task performed with and without tatami 
As for the variables associated with maturation, for bone age, we registered $13.3 \pm 1.8$ years and 13.3 \pm 1.9 years for boys and girls, respectively. For decimal age, the respective values were $12.8 \pm 1.8$ years and $13.0 \pm 1.5$ years. There was no significant difference in any of these variables between genders.

For the whole sample, bone age was significantly higher than decimal age, by 0.58 years $(Z=2.854$; $p<0.01$ ); these differences, however, did not occur between genders.

Figure 3 presents the proportions of the participants at each stage of maturation, by gender and practice floor.

There were no athletes with delayed maturation in either gender group. In the investigated sample, we registered $84.2 \%$ of the boys and $93.8 \%$ of the girls in the proper maturation stage. With regard to early maturing, males constituted the proportion of $15.8 \%$ and females that of $6.3 \%$.

Data also showed that the proper maturation stage was the most frequent among athletes who trained with tatami (92.6\%). The highest percentage of early maturation was observed in the group without tatami (18.5\% as compared with $7.4 \%$ in the tatami group).

Considering the frequencies of the variables of femur and wrist epiphyseal stage in relation to gender, it was verified that the stage of epiphyseal 'non union' had higher frequencies (> 63\%), with the exception of the epiphyseal plate of the female femur, which obtained higher frequency in the 'onset of union' stage
(44\%). There were no athletes in the 'complete union' epiphyseal stage of the femur or wrist in either gender. Also, no participants exhibited the 'recent union' stage for wrist in either gender. Females presented the highest frequencies in the following epiphyseal stages of the femur: 'onset of union' (43.8\%), 'active union' (18.8\%), and 'recent union' (12.4\%). This tendency was also confirmed for the wrist. There were no significant differences for femur epiphyseal growth stage $(U=197.500$; $p=0.27$ ) between males and females.

As for the frequencies of epiphyseal stages of femur and wrist depending on floor (Figure 4), one can observe a down-going trend from the first stage of 'non union' to the last stage of 'complete union'. It also appears that there was a trend with regard to the first stage of the femur values which shows that, when compared with the wrist, there were less subjects in the 'non union' stage.

For maturation variables, considering the athletes' training floor, there were no significant differences regarding bone age $(U=291.000 ; p=0.194)$, femoral epiphyseal stage $(U=313.000 ; p=0.328)$, wrist epiphyseal stage $(U=318.000 ; p=0.310)$, or maturation stage $(U=324.000 ; p=0.229)$.

A positive and moderate correlation was found between the years of practice and the femur epiphyseal stage in the tatami group $(\rho=0.429 ; p<0.05)$. The trend line was represented by $\mathrm{y}=0.2511 \mathrm{x}-0.2638$, and $\mathrm{R}^{2}=0.1823$ (Figure 5).
- Delayed maturation $(\mathrm{BA}<2 \mathrm{Cl})$ $\square$ Right maturation $(\mathrm{BA} \pm 2 \mathrm{Cl})$ Early maturation $(\mathrm{BA}>2 \mathrm{Cl})$
$\mathrm{BA}$ - bone age

$\mathrm{Cl}$ - confidence interval

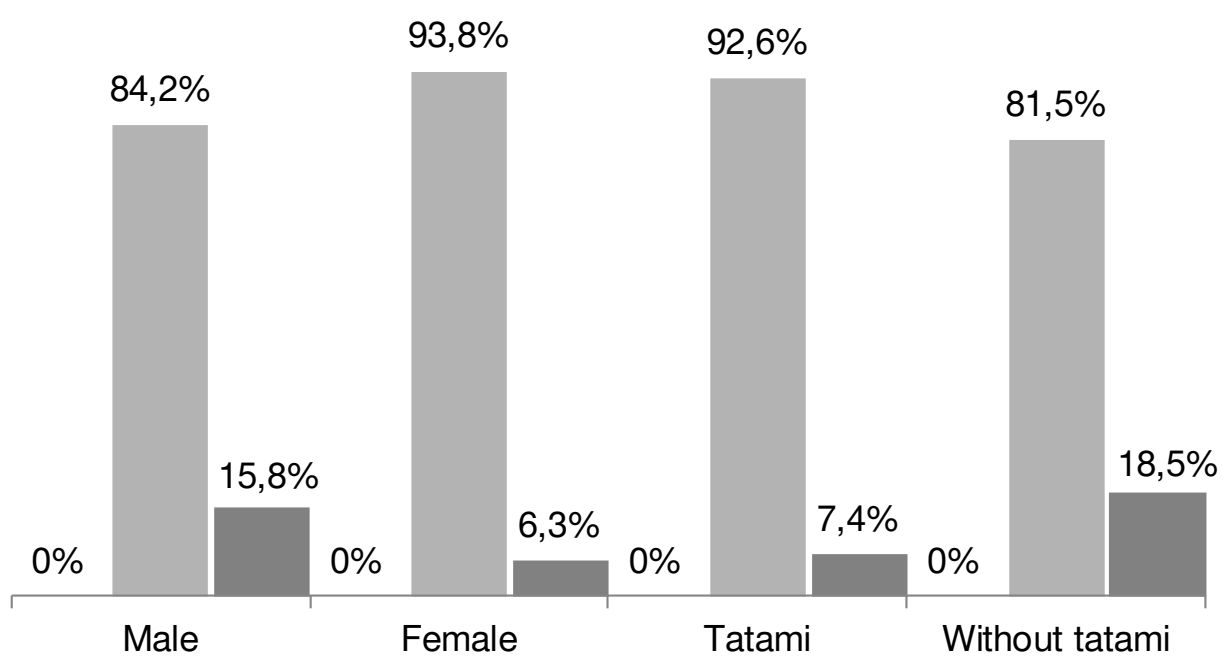

Figure 3. Frequency of maturation stages by gender and floor 


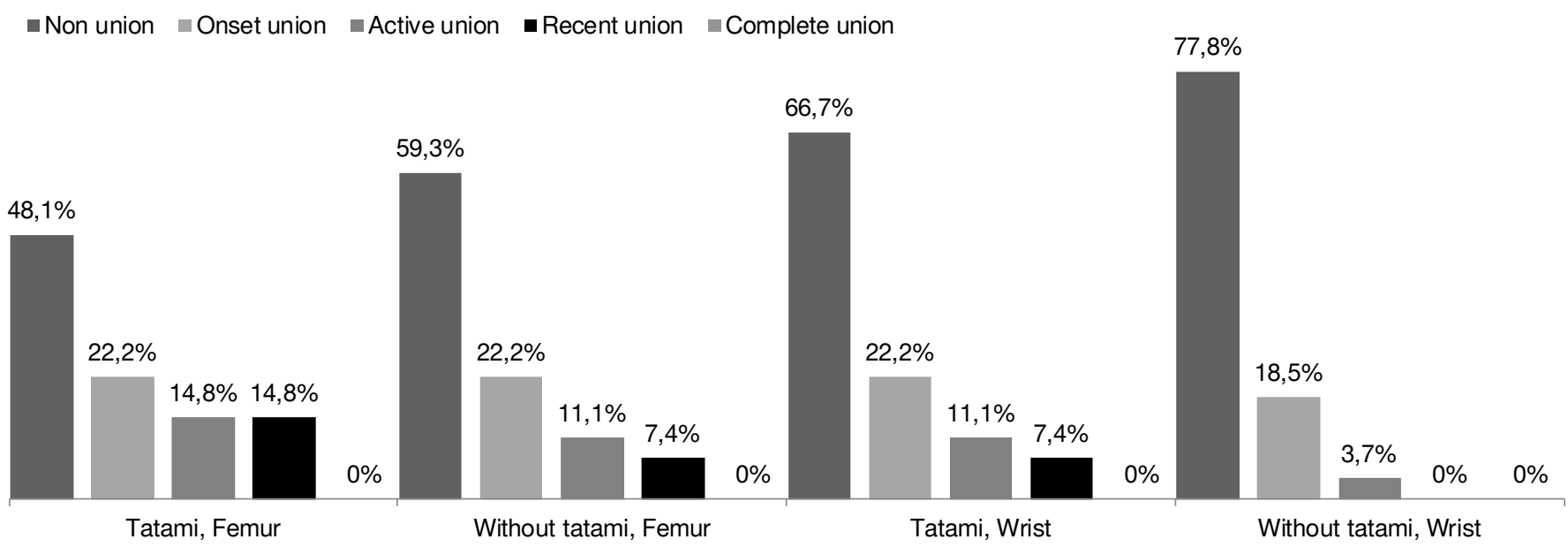

Figure 4. Frequencies of the epiphyseal stages of the femur and wrist per floor

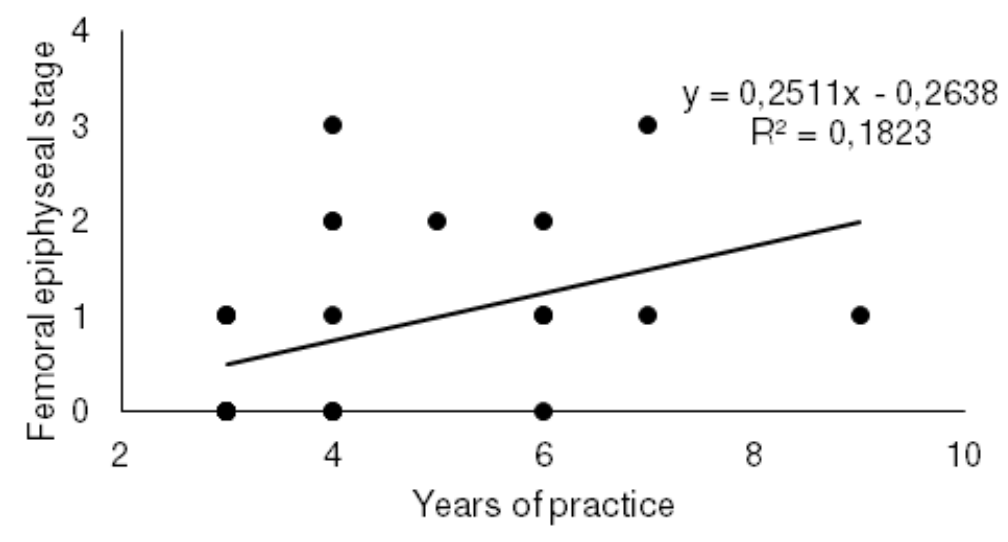

Figure 5. Correlation and trend line between years of practising taekwondo and the epiphyseal stage of the femur

\section{Discussion}

The aim of this study was to analyse and characterize the taekwondo GRFs and to relate them to specific maturation variables in order to verify if taekwondo practice with and without tatami appeared as a bio-positive or bio-negative factor for the maturation of the adolescent taekwondo athlete. In our study, the average of contacts per training or the average contacts per minute reveals a frequency within the biopositive limit that establishes high magnitude frequencies (3750-4050 contacts) during a 30-minute session [18]. The bio-positive effects include osteogenic stimulation, strengthening, growth and development of the musculoskeletal system, and enhancement of functional abilities at any age. On the basis of these results, it is suggested that taekwondo training does not generate bio-negative effects through the number of contacts. It is fundamental to evaluate the osteogenic potential of the tasks and the repeatability of the jumps in the sports activities since it could be a factor that could generate future injuries [19]. In this way, quantifying the mechanical load from GRFs in taekwondo and its links with the maturation of the adolescent athletes was an issue in this work; we found an average of $2.12 \mathrm{BW}$ for athletes practising on tatami and 2.02 BW for those who practised without tatami. These data are in agreement with the reference values of normalized GRFs, where a moderate intensity is 1-4 BW and low intensity magnitudes are below 1 BW [20]. In the context of these results, we verified that in this sample, the adolescent taekwondo athletes achieved GRF values that remained within the moderate range and that were considered a bio-positive stimulus.

There was a significant difference between absolute and normalized GRF values between the tatami and non-tatami practice. Even with the inclusion criterion of at least 3 years of taekwondo practice, this places the athletes at a good technical and developmental level in the sport, confirming that the magnitudes of the contacts depend on the type of surface, the landing final velocity, and the time of deceleration of this velocity - variables influenced by the strategy adopted by the athlete after contact with the floor and by such factors as body mass and the correct technique [21]. These latter factors aid the minimization of GRFs 
that act on the musculoskeletal system [22]. The results also prove that the magnitudes of GRF in taekwondo, with or without tatami, do not question the maturation parameters under study, which is in agreement with others investigations [10] that indicated no propensity among adolescent taekwondo athletes to have an abnormal maturation. Thus, it seems that taekwondo practice does not cause deformity when implemented in accordance with age and development and therefore does not constitute an injury risk factor [2].

The maturation stage achieved at the time of the data collection through bone indicators determines the rhythm of biological development and the relation of this event to the chronological age [23]. These variables did not show significant differences between the practice with and without tatami or between genders. However, athletes are classified into a suitable maturation according to the authors [24, 25].

Also with regard to the maturation stages, in both settings (with and without tatami), there were no significant differences, implying that the training with tatami and without tatami does not change the maturation of the athletes. This was evidenced by the absence of athletes in the delayed maturation stage. It is noteworthy that $18.5 \%$ of the athletes who practised without tatami were in the stage of early maturation, while only $7.4 \%$ of those training with tatami were at the proper stage of maturation. Although not proven in this study, this difference may be related to the effect of mechanical loading on the athlete's bone development, as suggested by some other authors [26-28] who reinforce the benefit and importance of regular training.

Considering that for the entire sample, most of the subjects were in the proper stage of maturation, we can say that we are facing a scenario where adolescent taekwondo athletes mature healthy and normal. This statement is in line with the findings by Nasri et al. [29], who concluded that moderate sports, particularly martial arts, benefited children and adolescents in maximizing their bone constitution.

Regarding the wrist and femoral epiphyseal plates, our study demonstrated no significant differences between genders. However, the stage of 'non union' had higher frequencies; an exception was the femoral epiphyseal plate in the girls, with a higher frequency in the early stage union (43.8\%). This is in agreement with Bontrager and Lampignano [30], indicating that the female skeleton usually becomes mature earlier than the male one and that the time for each bone to complete its growth varies depending on the body region, as demonstrated by the frequency of stage 0 ('non union') in the wrist of $76.3 \%$ for males and $62.5 \%$ for females.
These frequencies are superior to those found in the epiphyseal stages of the femur, which implies that there is a slower rate of maturation in the wrist.

The analysis of the femur and wrist epiphyseal stages among athletes training with and without tatami showed no significant differences. These results indicate that the floor does not change the maturation variables and does not pose a risk for the athletes [31].

The wrist epiphyseal plates presented a higher frequency of the first epiphyseal stages ('non union' and 'onset of union') as compared with the femur. This can be explained by the considerable frequency of actions with the lower limbs [1, 32-34], which has been associated with the development of osteogenic factors, and by increasing longitudinal bone growth and triggering changes in the structure of epiphyseal plates advancing their closure stages [28].

Analysing the practice with tatami, we verified that these athletes presented higher frequencies in more advanced stages of the epiphyseal plate than the individuals who trained without tatami. This indicates that the practice of taekwondo with tatami is related to the development of the epiphyseal plates and bone maturation without a detriment of body structure [2], suggesting that a possible abnormality in the maturation of some athletes is a consequence of an alteration of the epiphyseal plate due to a poor reactive bone formation [10]. This was not observed in the present study, which confirms that moderate sports, especially martial arts, benefit children and adolescents in maximizing their bone constitution [29]. As noted by O'Connor et al. [15], in this sample we also demonstrated that the higher the decimal age, the more advanced the epiphyseal stage of the femur.

This study presents a relevant contribution to the scientific knowledge and practical application of training in taekwondo, extensible to other martial arts whose traditional practice is performed without tatami. With this work, since the scientific evidence is not entirely sound on this subject, we encourage the scientific community to further study the issue. However, we reassure the coaches and athletes who for one reason or another still promote training barefoot and without tatami - the practice of taekwondo and martial arts benefits children and adolescents in maximizing their bone constitution.

Through the set of obtained results, it was verified and concluded that for this sample, the practice of taekwondo, regardless of whether it was performed with or without tatami, was advised owing to the bio-positive effect and osteogenic characteristics in the maturing bone. However, we can disclose some limitations 
F. Rocha, A. Conceição, C. Mata, J. Narciso, A.M. Costa, M. Branco, H. Louro, Reaction forces in taekwondo

in this work. For instance, bone mineral density, bone geometry, or bone quality were not examined: only radiologic investigation of the growth plate status was performed. These are therefore factors that should be addressed in a future study. In terms of methodology, the study should be powered to look for differences between males and females.

\section{Conclusions}

The frequency and amplitude of the reactive forces during taekwondo practice with and without tatami do not negatively influence the maturation process in young taekwondo practitioners.

\section{Acknowledgments}

The expert technical assistance of Dr. José Rianço Josué, Dr. Isabel Sapeiro, Dr. Carlos Maia Dias, and Dr. Pedro Silva in collecting and analysing data is kindly acknowledged and appreciated. The authors thank the athletes who reported to the laboratory for the evaluations. We also thank the Santarém District Hospital for the support of this study.

\section{Disclosure statement}

No author has any financial interest or received any financial benefit from this research.

\section{Conflict of interest}

The authors state no conflict of interest.

\section{Funding}

This study benefited from the support of national funding through the Portuguese Foundation for Science and Technology, I.P., under project UID04045/2021.

\section{References}

1. Lystad RP, Gregory K, Wilson J. The epidemiology of injuries in mixed martial arts: a systematic review and meta-analysis. Orthop J Sports Med. 2014;2(1):23259 67113518492; doi: 10.1177/2325967113518492.

2. Dos Santos SG, Detanico D, dos Reis DC. Magnitude of lower limb impact and kinematics in the handball jump throw [in Portuguese]. Rev Bras Cineantropom Desempenho Hum. 2009;11(3):326-333; doi: 10.1590/ 1980-0037.2009v11n3p326.

3. Devita P, Skelly WA. Effect of landing stiffness on joint kinetics and energetics in the lower extremity. Med Sci Sports Exerc. 1992;24(1):108-115; doi: 10.1249/00 005768-199201000-00018.

4. Bae Y-S, Kim K-M. Characteristic comparison of ground reaction force of the taekwondo's Apkubi motion and the walking on older persons. Korean J Sport Biomech. 2011; 21(3):289-296; doi: 10.5103/KJSB.2011.21.3.289.
5. Brennecke A, Amadio AC, Serrão JC. Dynamic parameters of selected movements of capoeira [in Portuguese]. Rev Port Cien Desp. 2005;5(2):153-159; doi: 10.5628/ rpcd.05.02.153.

6. Jandačka D, Estevan I, Janura M, Falco C. The impact of the initial stance position on lower limb joint kinetics in the taekwondo roundhouse kick. Acta Univ Palacki Olomuc Gymn. 2013;43(2):15-22; doi: 10.5507/ag.2013. 008.

7. Alves C, Lima RVB. Linear growth and puberty in children and adolescents: effects of physical activity and sports [in Portuguese]. Rev Paul Pediatr. 2008;26(4): 383-391; doi: 10.1590/S0103-05822008000400013.

8. Caine D, DiFiori J, Maffulli N. Physeal injuries in children's and youth sports: reasons for concern? Br J Sports Med. 2006;40(9):749-760; doi: 10.1136/bjsm.2005. 017822.

9. Da Silva Filho JN, Cordeiro RCFSC, Cruz AP, Filho JRMG, Saraiva B, Ferreira RA. Effects of strength exercise on bone development in children and adolescents: a systematic review [in Portuguese]. Rev Bras Prescr Fisiol Exerc. 2015;9(51):40-47.

10. Siebenrock KA, Behning A, Mamisch TC, Schwab JM. Growth plate alteration precedes cam-type deformity in elite basketball players. Clin Orthop Relat Res. 2013; 471(4):1084-1091; doi: 10.1007/s11999-012-2740-6.

11. Fuchs RK, Smow CM. Gains in hip bone mass from high-impact training are maintained: a randomized controlled trial in children. J Pediatr. 2002;141(3):357362; doi: 10.1067/mpd.2002.127275.

12. Ross WD, Marfell-Jones MJ. Kinanthropometry. In: MacDougall JD, Wenger HA, Green HJ (eds.), Physiological testing of the high-performance athlete. Champaign: Human Kinetics Books; 1991; 223-308.

13. Barela AMF, Duarte M. Use of force plate for acquisition of kinetic data during human gait [in Portuguese]. Braz J Mot Behav. 2011;6(1):56-61; doi: 10.20338/ bjmb.v6i1.32.

14. Leong JY, Wong JE. Accuracy of three android-based pedometer applications in laboratory and free-living settings. J Sports Sci. 2017;35(1):14-21; doi: 10.1080/ 02640414.2016 .1154592$.

15. O’Connor JE, Bogue C, Spence LD, Last J. A method to establish the relationship between chronological age and stage of union from radiographic assessment of epiphyseal fusion at the knee: an Irish population study. J Anat. 2008;212(2):198-209; doi: 10.1111/j.14697580.2007.00847.x.

16. Greulich WW, Pyle SI. Radiographic atlas of skeletal development of the hand and wrist, $2^{\text {nd }}$ ed. Stanford: Stanford University Press; 1959.

17. Tanner JM, Healy MRJ, Goldstein H, Cameron N. Assessment of skeletal maturity and prediction of adult height (TW3 method). London: WB Saunders; 2001.

18. Nigg BM, Cole GK, Brüggemann GP. Impact forces during heel-toe running. J Appl Biomech. 1995;11(4):407432. 
19. Coelho GFM, dos Santos SG. Characterization of impact forces for implementing of jump kick [in Portuguese]. Mot Rev Educ Fis. 2010;16(1):143-151; doi: 10.5016/ 1980-6574.2010v16n1p143.

20. Nigg BM, MacIntosh BR, Mester J. Biomechanics and biology of movement. Champaign: Human Kinetics; 2000.

21. Abilel BS, Serrão JC, Sacco ICN, Amadio AC. Dynamic, kinematic, and electromyographic characteristics of landings in expected and unexpected situations [in Portuguese]. Rev Bras Biomec. 2002;3(4):45-50.

22. McGinnis PM. Biomechanics of sport and exercise, $3^{\text {rd }}$ ed. Champaign: Human Kinetics; 2013.

23. Beunen GP, Rogol AD, Malina RM. Indicators of biological maturation and secular changes in biological maturation. Food Nutr Bull. 2006;27(4):244-256; doi: 10.1177/15648265060274S508.

24. Malina RM, Bouchard C, Bar-Or O. Growth, maturation, and physical activity, $2^{\text {nd }}$ ed. Champaign: Human Kinetics; 2004.

25. Rêgo C. Physical or sexual maturation and bone maturation in males [in Portuguese]. Rev Med Desportiva Informa. 2011;2(6):23-25.

26. Larsen MN, Nielsen CM, Helge EW, Madsen M, Manniche V, Hansen L, et al. Positive effects on bone mineralisation and muscular fitness after 10 months of intense school-based physical training for children aged 8-10 years: the Fit First randomised controlled trial. Br J Sports Med. 2018;52(4):254-260; doi: 10.1136/ bjsports-2016-096219.

27. Da Silva CC, Teixeira AS, Goldberg TBL. Sport and its implications on the bone health of adolescent athletes. RevBras MedEsporte. 2003;9(6):433-438; doi: 10.1590/ S1517-86922003000600007.

28. Yung PS, Lai YM, Tung PY, Tsui HT, Wong CK, Hung VWY, et al. Effects of weight bearing and non-weight bearing exercises on bone properties using calcaneal quantitative ultrasound. Br J Sports Med. 2005;39(8): 547-551; doi: 10.1136/bjsm.2004.014621.

29. Nasri R, Hassen Zrour S, Rebai H, Neffeti F, Najjar MF, Bergaoui N, et al. Combat sports practice favors bone mineral density among adolescent male athletes. J Clin Densitom. 2015;18(1):54-59; doi: 10.1016/j.jocd.2013. 09.012.

30. Bontrager KL, Lampignano JP. Textbook of radiographic positioning and related anatomy. St. Louis: Elsevier; 2013.

31. Melo SIL, dos Santos SG, Cinelli MJ, Borges Junior NG, Heidrich RM. Mechanical characteristics of tatami used in judo [in Portuguese]. Rev Bras Educ Fis. 2004;18(3): 227-238; doi: 10.1590/S1807-55092004000300002.

32. Beis K, Pieter W, Abatzides G. Taekwondo techniques and competition characteristics involved in time-loss injuries. J Sports Sci Med. 2007;6:45-51.
33. Lystad RP, Pollard H, Graham PL. Epidemiology of injuries in competition taekwondo: a meta-analysis of observational studies. J Sci Med Sport. 2009;12(6):614621; doi: 10.1016/j.jsams.2008.09.013.

34. Pieter W, Fife GP, O’Sullivan DM. Competition injuries in taekwondo: a literature review and suggestions for prevention and surveillance. Br J Sports Med. 2012; 46(7):485-491; doi: 10.1136/bjsports-2012-091011. 